\title{
1 Genetic Characterization and Curation of Diploid A-Genome Wheat Species
}

3 Laxman Adhikari ${ }^{1,2}$, John Raupp ${ }^{1}$, Shuangye Wu ${ }^{1}$, Duane Wilson ${ }^{1}$, Byron Evers ${ }^{1}$, Dal-Hoe Koo $^{1}$,

4 Narinder Singh ${ }^{1, *}$, Bernd Friebe ${ }^{1}$ and Jesse Poland ${ }^{1,2, *}$

6 Affiliations:

$7 \quad{ }^{1}$ Department of Plant Pathology, Kansas State University, Manhattan, KS; Wheat Genetic

8 Resource Center (WGRC), Kansas State University, Manhattan, KS 66502

$9{ }^{2}$ Center for Desert Agriculture, King Abdullah University of Science and Technology, Thuwal,

10 Saudi Arabia

$11 *$ current address: Bayer Crop Science

12 *Corresponding author: jpoland@ksu.edu, jesse.poland@kaust.edu.sa

14 The author responsible for distribution of materials integral to the findings presented in this

15 article in accordance with the policy described in the Instructions for Authors

16 (https://academic.oup.com/plphys/pages/General-Instructions) is Laxman Adhikari_.

Short title: Characterization of Diploid Wheat

One-sentence summary: Genotyping diploid A-genome relatives of wheat uncovered high genetic diversity and unique evolutionary relationships giving insight to the effective use of this germplasm for wheat improvement.

\section{Author Contributions:}

25 JP and JR designed the experiment and conceptualized the study. JR, SW, DW, BE carried out 26 experiments and data collection, growing of plant materials and germplasm. DHK performed

27 cytological work. LA conducted computational analysis and phenotypic data collection. NS assisted in computational work. JP and BF supervised the experiment. LA and JP wrote the manuscript. All the authors have read and approved the manuscript for publication. 


\section{Abstract}

33 A-genome diploid wheats represent the earliest domesticated and cultivated wheat species in the

34 Fertile Crescent and include the donor of the wheat A sub-genome. The A-genome species

35 encompass the cultivated einkorn (Triticum. monococcum L. subsp. monococcum), wild einkorn

36 (T. monococcum L. subsp. aegilopoides (Link) Thell.), and T. urartu. We evaluated the

37 collection of 930 accessions in the Wheat Genetics Resource Center (WGRC) using genotyping-

38 by-sequencing (GBS) and identified 13,860 curated SNPs. Genomic analysis detected misclassified and genetically identical (> 99\%) accessions, with most of the identical accessions originating from the same or nearby locations. About $56 \%(n=520)$ of the WGRC A-genome

41 species collections were genetically identical, supporting the need for genomic characterization

42 for effective curation and maintenance of these collections. Population structure analysis

43 confirmed the morphology-based classifications of the accessions and reflected the species

44 geographic distributions. We also showed that T. urartu is the closest A-genome diploid to the

45 A-subgenome in common wheat (Triticum aestivum L.) through phylogenetic analysis.

46 Population analysis within the wild einkorn group showed three genetically distinct clusters,

47 which corresponded with wild einkorn races $\alpha, \beta$, and $\gamma$ described previously. The $T$. monococcum genome-wide $\mathrm{F}_{\mathrm{ST}}$ scan identified candidate genomic regions harboring a

49 domestication selection signature at the non-brittle rachis 1(Btrl) locus on the short arm of 50 chromosome $3 \mathrm{~A}^{\mathrm{m}}$ at $\sim 70 \mathrm{Mb}$. We established an A-genome core set (79 accessions) based on 51 allelic diversity, geographical distribution, and available phenotypic data. The individual species 52 core set maintained at least $80 \%$ of allelic variants in the A-genome collection and constitute a 53 valuable genetic resource to improve wheat and domesticated einkorn in breeding programs.

Key Words: Einkorn, A-genome wheat species, T. urartu, T. monococcum, aegilopoides, 57 genetically identical, population structure, Nei’s index, F $F_{S T}$ scan, Btrl, selection signal, GBS, misclassified, core collection 
62 Wheat wild relatives are an important reservoir of genetic diversity that can be utilized for wheat

63 improvement, particularly for diseases, insect pests, and abiotic stress tolerance (Wulff and

64 Moscou, 2014). Cultivated tetraploid (pasta wheat, Triticum turgidum) and hexaploid (bread wheat, Triticum aestivum L.) wheat arose through successive whole-genome hybridization between related species in the Triticeae. Although polyploidization in wheat enabled broad adaptation and genome plasticity found in polyploids (Comai, 2005), it also created severe genetic bottlenecks within each subgenome (Feldman and Levy, 2012). Likewise, of the three natural races within wild einkorn, only one natural race $(\beta)$ has been domesticated, thus, genetic diversity in the wild einkorn is expected to be greater than in domesticated einkorn (Pourkheirandish et al., 2018). Some recent findings, however, reported no or low reduction in

72 nucleotide diversity through einkorn domestication, most likely indicating a minimal bottleneck

73 during domestication of cultivated einkorn (Kilian et al., 2007). This was true when diversity comparisons were performed between wild einkorn specific races ( $\alpha$ and $\beta$ ) vs. domesticated einkorn. However, when the comparison was made between the domesticated einkorn vs. all groups of wild einkorn, the wild einkorn diversity was much higher than found in the cultivated accessions. The value of A-genome species diversity for alleviating the wheat diversity bottleneck has been described (Brunazzi et al., 2018; Mondal et al., 2016). Thus, diversity assessment in germplasm collections of diploid A-genome species is crucial for conservation planning and efficient utilization of germplasm in breeding. A-genome wheat species $(2 \mathrm{n}=2 \mathrm{x}=14, \mathrm{AA})$ are diploid grasses including the wild einkorn $(T$. monococcum L. subsp. aegilopoides (Link) Thell.), domesticated einkorn (T. monococcum L. subsp. monococcum), and T. urartu (van Slageren, 1994). Molecular and cytological studies have confirmed that $T$. urartu, a related species sharing the same genome as domesticated einkorn, is the A-genome ancestor to cultivated wheat (T. aestivum) (Dvorak et al., 1988; Dong et al., 2012). In the first polyploidization event that occurred $~ 0.5-0.15$ million years ago (MYA) (Charmet, 2011), T. urartu naturally hybridized with a B-genome donor grass, an extant species but close relative of Aegilops speltoides Tausch, giving rise to the wild tetraploid wheat T. turgidum L. 
91 the next event, the cultivated tetraploid emmer wheat (T. turgidum subsp. durum (Desf.) Husn.)

92 naturally hybridized with the D-genome donor species (Ae. Tauschii Coss) forming hexaploid

93 bread wheat (AABBDD, $2 n=6 x=42)$. The A-genome species morphologically resemble

94 cultivated tetraploid and hexaploid wheat more than any other surviving diploid wheat species

95 and are predominant in the Fertile Crescent (Heun et al., 1997). Domestication of einkorn wheat,

96 together with emmer wheat and barley around 12,000 years ago, transformed human culture

97 from hunting-gathering to agriculture, popularly known as the 'Neolithic Revolution' (Kilian et

98 al., 2010). The Karacadağ mountain in the southeast Turkey has been considered the

99 geographical point for einkorn domestication (Brandolini et al., 2016).

100

101 The donor of the A genome of the bread wheat, $T$. urartu, is estimated to have diverged nearly

1020.57 - 0.76 MYA from another widespread A-genome diploid species, T. monococcum.

103 Interspecific crosses between T. urartu and T. monococcum are infertile, confirming the large

104 phylogenetic distance and genetic differentiation of the species (Middleton et al., 2014). Like

105 hexaploid wheat, A-genome species have a large genome size with a mean nuclear DNA content

106 of $5.784 \mathrm{pg} / 1 \mathrm{C}$ in T. urartu to $6.247 \mathrm{pg} / 1 \mathrm{C}$ in T. monococcum subps. aegilopoides (Özkan et al.,

107 2010). Morphologically, T. urartu possesses smooth leaves, a brittle rachis, and smaller anthers

108 (<0.3 mm). The wild einkorn (T. monococcum subsp. aegilopoides) are characterized with a

109 brittle rachis, hairy leaves, and larger $(\geq 0.5 \mathrm{~mm})$ anthers. Domesticated einkorn has a nonbrittle

110 (semi tough) rachis with smooth leaves (Brandolini and Heun, 2019).

112 Being homologous to the wheat A-genome, these species provide useful sources for wheat

113 improvement using wide crosses and cytogenetics approaches. The A-genome species are

114 important genetic resources for pest resistance and stress tolerance. For example, T. urartu was

115 identified as a source of resistance to the root lesion nematode Pratylenchus thornei (Sheedy et

116 al., 2012) and stem rust (Rouse and Jin, 2011). Novel stem rust resistance genes SrTm5 and Sr60

117 were mapped in an $\mathrm{F}_{2}$ population derived from crosses between wild and the cultivated einkorn

118 (Chen et al., 2018). Sr35, the first gene cloned against the devastating stem rust race UG99, also

119 originates from T. monococcum (Saintenac et al., 2013). A leaf rust gene, Lr63, in wheat

120 chromosome 3AS was introgressed from T. monococcum (Kolmer et al., 2010). Surveying the

121 genetic variation in A-genome species that can be utilized in wheat improvement has lagged, 
122 considering the potential value of more effectively utilizing these species for wheat

123 improvement.

125 Einkorn has multiple botanical names in the literature as proposed by the various taxonomists, 126 and confusion related to the einkorn nomenclature is widespread. In 1948, Schiemann classified 127 einkorn as wild einkorn (T. boeoticum subsp. thaoudar), the feral einkorn (T. boeoticum subsp. 128 aegilopoides), and the domesticated einkorn (T. monococcum subsp. monococcum) (Schiemann, 129 1948; Brandolini et al., 2016). Mac Key published einkorn classification in 1954 (Mac Key, 130 1954) and updated the nomenclature several times through 2005 (Mac Key, 2005a). van Slageren 131 also published the einkorn nomenclature, where the wild and domesticated einkorn were simply 132 named as T. monococcum L. subsp. aegilopoides (hereafter subsp. aegilopoides) and T. 133 monococcum L. subsp. monococcum (hereafter subsp. monococcum), respectively (van Slageren, 134 1994). In this study, we follow van Slageren (1994) einkorn taxonomy, because the A-genome 135 species collection in the Wheat Genetics Resource Center (WGRC) at Kansas State University 136 (KSU) were initially classified using this nomenclature (van Slageren, 1994).

138 A well-characterized population structure of A-genome species is critical to formulating 139 effective conservation strategy, selecting diverse germplasm, and enhancing the accuracy of the 140 genomic analysis with structure information (Singh et al., 2019). Population structure and 141 diversity assessment have become easier with next-generation sequencing, which makes

142 discovery of thousands of genotyping markers possible. Here, we used genotyping by sequencing 143 (GBS) for single nucleotide polymorphism (SNP) discovery. GBS is straightforward, high144 throughput, and with multiple downstream pipelines for data processing (Poland et al., 2012a).

145 However, population structure of A-genome species has not been evaluated in detail with the 146 resource of whole-genome profiling. Therefore, our objectives are to: i) curate A-genome wheat 147 accessions in the gene bank by recognizing genetically identical and misclassified accessions, ii) 148 assess the population structure and genetic diversity of the A-genome wheat species, and iii) 149 establish genetically, geographically, and phenotypically representative core collections for A150 genome species within the WGRC gene bank. 


\section{Results}

\section{A-Genome Species Distribution}

156 Most of the wild einkorn (subsp. aegilopoides) in our collection, were collected across Turkey,

157 northern Iraq, west Iran, and Transcaucasia, whereas the majority of domesticated einkorn

158 (subsp. monococcum) were from west Turkey and the Balkans (Figure 1, Supplemental Table

159 S1). About half of the T. urartu accessions were from eastern Lebanon, around the Beqaa Valley,

160 and a major part were from southeast Turkey (Figure 1). The A-genome species are known to

161 span from Transcaucasia through Anatolia to the Caspian Sea. Thus, the WGRC collection

162 generally covers the geographic range of this species. After genomic characterization including

163 misclassified accessions adjustment, we retained 196 T. urartu accessions, 145 domesticated

164 einkorn accessions, and 584 wild einkorn accessions (Supplemental Table S1). There were also 5

165 tetraploids identified in the population which were curated to correct species designations.

\section{Markers and Genotyping}

For all A-genome accessions, we identified 44,215 biallelic SNPs after a filter for passing Fisher exact test of disassociated alleles (doi:10.5061/dryad.9zw3r22f6). Upon filtration (MAF > 0.01, missing $<30 \%$, heterozygosity $<10 \%$ ) and species separation, we retained 13,860 SNPs in total comprising 3840 SNPs for T. urartu, 8989 SNPs for T. monococcum, 8283 SNPs for subsp.

172 aegilopoides, and 4401 for subsp. monococcum (Table 1). We observed loci that were fixed or 173 otherwise a single heterozygous genotype call within the individual species and subspecies. To

174 compute total segregating loci per group and minimize the effect of potential sequencing error,

175 we further filtrated and removed any loci that had only a single accession with heterozygous

176 genotype and were otherwise fixed in remaining population, if the population size was greater

177 than 100. For A-genome species diversity assessment, thousands of segregating loci were

178 available for the groups defined by population analysis and core set selections (Table 1). For

179 wheat and A-genome diploids together we found 15,300 filtered SNPs and used the information

180 to see specific relationships between the species.

\section{Gene Bank Curation}


183 We identified and corrected a total of 22 misclassified accessions using fastStructure analysis,

184 phylogenetic (Supplemental Figure S1) and PCA clustering including nine T. urartu, two

185 subsp. monococcum, six subsp. aegilopoides, and five tetraploid accessions. As a large number

186 of accessions in both T. urartu and subsp. aegilopoides were from southeast Turkey, we

187 observed most of the misclassified accessions also were from the same site (Supplemental Tables

188 S1 and S2).

190 While evaluating the collection for genetically identical accessions, we compared various

191 number of loci for allele matching per A-genome species (Table 2) as the SNPs were filtered to

192 keep only the sites with MAF > 0.05, less than 50\% missing, and less than 10\% heterozygous.

193 We identified and used a threshold of $\geq 99 \%$ identity by state (IBS) to declare the individuals as

194 genetically identical accessions (Supplemental Figure S2) with tolerance for sequencing and

195 genotyping error. With these criteria we identified a total of $520(56 \%)$ accessions within the

196 collection having at least one other accession that was genetically identical. These identical

197 accessions were mostly observed within T. urartu (135) and within $\alpha$ race subsp. aegilopoides

198 (326) (Table 2 and Supplemental Table S1). To confirm this analysis, we checked the collection

199 sites of the groups of genetically identical accessions and identified that all of the respective

200 matching accessions were collected from the same or nearby sites. We further observed the

201 identical accessions had matching phenotypes such as glume color, for which the scores were the

202 same for all accessions within the respective sets of identical accessions (Supplemental Table

203 S1). For instance, accession no. TA471 had 11 other identical accessions which all had glume

204 color score of 7 on a scale of 1 (white) to 9 (black) (Supplemental Table S1). This further

205 confirmed the utility and accuracy of using the GBS data for identification of genetically

206 identical accessions.

207

208 Relationship Between A-genome Diploid and Wheat

209 The genetic grouping of A-genome diploids and CIMMYT wheat lines together showed that

210 wheat is closer to T. urartu than to T. monococcum (Supplemental Figure S3), a finding in

211 agreement with the known relationship between the species. The unrooted NJ tree constructed

212 for wheat and A-genome diploid wheat showed five accessions (TA282, TA10915, TA1325,

213 TA1369, and TA10881) clustering far from the T. urartu major clade (Supplemental Figure S3). 
214 Cytological analysis identified them as tetraploid $(2 n=28)$ (Supplemental Figure S4). Therefore,

215 we corrected the species designations for these five accessions and excluded them from further

216 population analysis. This observation confirms that GBS also enables identifying cryptic

217 accessions with different ploidy levels in the population.

219 A-genome Population Structure and Wild Einkorn Genetic Races

220 Population grouping in the fastStructure analysis at $K=2$ to $K=7$ showed the A-genome genetic 221 structure was split with the known biological and geographical characterization (Figure 2). This

222 analysis revealed a number of misclassified accessions that were individually curated and 223 checked, including morphological confirmation, and were reclassified to the appropriate group 224 (Supplemental Table S2).

226 At $\mathrm{K}=2$, the population differentiation occurred only at the level of species, the accessions split 227 into T. monococcum and T. urartu, confirming known species differences (Figure 2). At $\mathrm{K}=3$, the 228 two subspecies of T. monococcum differentiated with the accessions in the $\alpha$ wild einkorn race 229 were clearly differentiated from domesticated einkorn. However, the other races of wild einkorn 230 ( $\beta$ and $\gamma$ ) appeared to be an admixture, supporting that there is incomplete differentiation 231 between the wild and domesticated einkorn, a classification that is simply based on the few 232 morphological characteristics of the domestication syndrome.

234 We observed differentiation of wild einkorn into genetically distinct groups at $\mathrm{K}=4$. Comparing 235 these three wild einkorn subgroups with the $\alpha, \beta$, and $\gamma$ wild einkorn races described by (Kilian et 236 al., 2007), we reported the three genetic subgroups as representing the races $\alpha, \beta$, and $\gamma$ by 237 identifying common USDA Plant Introduction (PI) numbers for accessions in both studies. The 238 genetic clustering pattern and geographical distribution then confirmed that the subgroups within 239 subsp. aegilopoides represents $\alpha, \beta$, and $\gamma$ races described and we hereby name these genetic 240 groups accordingly (Supplemental Table S3) (Kilian et al., 2007). In Kilian et al. (2007), the $\alpha$ 241 race accessions were primarily from southeast Turkey, northern Iraq, and Iran; the $\gamma$ race 242 involves accessions from Transcaucasia to western Anatolia; and the $\beta$ race comprises a few 243 accessions collected around Karacadag Turkey (Figure 1, Supplemental Table S1). Based on 244 population differentiation, $\alpha$ race exhibited the strongest differentiation with domesticated 
245 einkorn and should represent the base population of subsp. aegilopoides, whereas the $\beta$ race of

246 wild einkorn exhibited the least differentiation with subsp. monococcum. Interestingly, the $\beta$ race

247 did not fully differentiate from subsp. monococcum at any number of K (Figure 2), (1)

248 supporting that domesticated einkorn originated out of this subpopulation, which already largely

249 differentiated from the other wild einkorn, or (2) that the $\beta$ race represents 'feral' subsp.

250 monococcum accessions that were, at one point, fully domesticated but reverted to wild plant

251 types through introgression and admixture.

253 At $\mathrm{K}=5$, the population subgrouping according to the accession origin was observed in $\alpha$ race

254 accessions within the wild einkorn. Accessions from Erbil (ancient name 'Arbil') differentiated

255 as a subpopulation, and the accessions from Sulaymaniyah (Iraq) split as the admixture of the

256 Erbil subgroup and the remaining accessions at $\mathrm{K}=5$ (Figure 2). We could not observe any new

257 differentiation within the wild einkorn group at $\mathrm{K}=6$. However, at $\mathrm{K}=7$, we observed three

258 distinct subgroups and a higher level of admixture within the $\alpha$ race of subsp. aegilopoides

259 (Figure 2). Also, there were two main sets of admixture types; the first set mainly consists of

260 accessions from Iran that shared ancestry from the Duhok and Turkey subgroups, and the second

261 corresponds with accessions from Sulaymaniyah (Iraq) and has ancestry from all three

262 subgroups. Hence, within the population of $\alpha$ race einkorn accessions, three subgroups exist;

263 Erbil, Duhok, and Turkey, and two groups of genetic admixtures (Iran and Sulaymaniyah),

264 named from their origin.

266 We did not observe any subgrouping within the accessions from the southeast Turkey, yet the 267 accessions were primarily from two sites (Sanliurfa and Mardin). The grouping pattern of three 268 subgroups within the $\alpha$ race accessions provided an insight into the wild einkorn subgrouping 269 and their genetic relationships. We did not observe within population differentiation in 270 domesticated einkorn group.

272 In $T$. urartu, the subgrouping occurred at $\mathrm{K}=6$, and was unchanged at $\mathrm{K}=7$ (Figure 2). Two major

273 T. urartu subgroups represented accessions from Turkey (\#T) and another from Lebanon (\#L).

274 Few T. urartu accessions were from Syria (\#S); some showed admixture, and some had a clean

275 ancestry that resembled accessions from Turkey (Figure 2). The few remaining accessions 
276 primarily were from Transcaucasia (\#M) and exhibited an ancestry similar to accessions from

277 Turkey (Figure 2).

\section{Phylogenetic Clustering and PCA}

280 The phylogenetic clustering split the A-genome accessions into separate clades for T. urartu, T. monococcum subsp. monococcum, and all races within the subsp. aegilopoides (Figure 3). Only

28212 accessions were retained within race $\beta$, and the accessions were clustered with some other

283 domesticated einkorn accessions (Figure 3). The T. urartu clade distantly clustered in

284 phylogenetic analysis from either of the einkorn clade indicating the obvious genetic differences

285 between species. The misclassified accessions (Supplemental Figure S1) observed in the

286 phylogenetic clustering were re-classified into proper genotype-based classes.

A PCA plot of A-genome species also showed accessions clustering as in fastStructure and phylogenetic analysis (Supplemental Figure S5). The first principal component (PC1), which grouped the accessions of T. monococcum and T. urartu in two primary clusters, explained 58\%

291 of the variation. The PC2, which divided the einkorn accessions, explained $8 \%$ of the variation

292 and separated domesticated and different races within the wild einkorn. Misclassified accessions

293 previously observed also were revealed in the PCA analysis and their taxonomy classification 294 adjusted.

\section{Genetic Diversity and F $_{\text {ST }}$}

297 A considerably high Nei's diversity index (0.25) was observed for the complete set of A-genome 298 accessions. The Nei's diversity indices for individual A-genome species ranged from 0.062 for 299 domesticated einkorn to 0.106 for the entire einkorn group. Among the three races of wild 300 einkorn, the Nei's diversity indices of $\beta$ race (0.065) was the lowest and $\gamma$ was the highest $(0.095$; 301 Table 1). As expected for diverse accessions, we found a high density of alleles with low minor 302 allele frequency (MAF) (Supplemental Figure S6).

304 Population differentiation within the A-genome species were further verified by pairwise fixation 305 index $\left(\mathrm{F}_{\mathrm{ST}}\right)$ values (Nei, 1987) computed between the groups. Pairwise $\mathrm{F}_{\mathrm{ST}}$ between T. urartu and entire einkorn were greater than 0.80 , supporting that the two species are strongly differentiated 
307 (Table 3). The pairwise $\mathrm{F}_{\mathrm{ST}}(0.56)$ between the $\alpha$ race and domesticated einkorn indicated the 308 strongest differentiation between any two groups within the einkorn, whereas the weakest 309 differentiation $\left(\mathrm{F}_{\mathrm{ST}}=0.31\right)$ was between the $\beta$ race and domesticated einkorn, supporting the 310 model that this wild race was the most likely forerunner of domesticated einkorn as previously

311 hypothesized (Kilian et al., 2007). The concept also was endorsed by the origin of $\beta$ race einkorn 312 in the WGRC collection, mostly from Diyarbakir and Sanliurfa, which are near Karacadag and 313 Kartal-Karacadag mountains - the likely points of domestication. Nonetheless, the genetic 314 grouping of $\beta$ also occurred with subsp. monococcum in the unrooted NJ tree (Figure 3).

315 Pairwise $\mathrm{F}_{\mathrm{ST}}(\sim 0.40)$ between pairs: ' $\gamma$ race - subsp. monococcum' and ' $\gamma$ race - $\alpha$ race' implicit

316 the differentiation of $\gamma$ race as a genetically intermediate type from truly wild $\alpha$ race and

317 domesticated einkorn (Table 3). The pairwise $\mathrm{F}_{\mathrm{ST}}$ computed between two subpopulations

318 (Turkey and Lebanon) of T. urartu was 0.52, which also agrees with the population structure 319 analysis.

321 Pairwise $\mathrm{F}_{\mathrm{ST}}$ computed between the subpopulations within $\alpha$ race of subsp. aegilopoides signaled

322 the geographical differentiation and the potential gene flow within this wild einkorn race.

323 Consistent with the fastStructure output, the Erbil subgroup showed the stronger differentiation 324 (higher $\mathrm{F}_{\mathrm{ST}}$ ) with other wild einkorn subgroups (Supplemental Table S5). The subgroup Duhok 325 and southeast Turkey and their admixture group (Iran) had the minimum pairwise $\mathrm{F}_{\mathrm{ST}}(\sim 0.12)$.

326 The accessions within the admixture group of Sulaymaniyah displayed almost similar

327 differentiation $\left(\sim \mathrm{F}_{\mathrm{ST}}=0.16\right)$ with three subgroups, which agrees with population structure as the 328 admixture group has ancestry from all three.

\section{$330 \quad$ F $_{\text {ST }}$ Scan and Einkorn Selection Signature}

331 After filtration and imputation, we had 6,622 SNPs segregating in T. monococcum on which we 332 calculated per site $\mathrm{F}_{\mathrm{ST}}$ values for each of the seven chromosomes that ranged from near 0 to 1.

333 Both methods, Porto-Neto et al. (2013) and VCFtools, produced similar results for raw and 334 smoothed $\mathrm{F}_{\mathrm{ST}}$ values. We used a genome-wide threshold of $3 \sigma(0.24)$ over the mean $\mathrm{F}_{\mathrm{ST}}$, from 335 which we observed only a single-selection signature on short arm of chromosome $3 \mathrm{~A}$ 336 (Supplemental Figure S7) after smoothing using Lowess method ( $f=0.1$ ) (Pintus et al., 2014). 337 This selection signature corresponded to the locus that harbors the non-brittle rachis 1 (Btrl) 
338 (Pourkheirandish et al., 2018) and was supported by the BLAST hit of a coding sequence

339 (Supplemental Text S1) of Btrl on the reference genome used to genotype our population $(T$.

340 urartu pseudomolecule), which was occurred at $62 \mathrm{Mb}$ on chromosome $3 \mathrm{~A}$. We also observed

341 that the raw $F_{\mathrm{ST}}$ values for three consecutive sites of the region $(62 \mathrm{Mb})$ had the highest $\left(\mathrm{F}_{\mathrm{ST}}=1\right)$

342 values. Thus, this selection scan identified the impact of strong selection for Btrl in the

343 domesticated einkorn.

\section{A-genome Core Collection}

346 To maximize the utility of the WGRC collection we identified a core set that captured the 347 majority of total allelic diversity within 19 T. urartu accessions, and 60 accessions of $T$. 348 monococcum (Supplemental Table S4). In core sets of the entire A-genome collection, we 349 captured $\sim 98 \%$ of the identified alleles (Figure 4), whereas each separate sub-core also captured 350 at least $79 \%$ of the segregating alleles of the respective species-specific collections (Table 1). 351 Enriching allelic diversity within the smaller core collections was confirmed by the higher Nei's 352 diversity index (0.26) of the selected core sets relative to the entire collection (0.25) (Table 1$)$.

353 Distribution of the core set accessions in the phylogenetic cluster, PCA clusters, and in the 354 geographic map showed that the selected accessions also represented all subgroups within the 355 population and covered the geographic range (Supplemental Figure S8 - S10). Ranges of glume 356 color scores (Supplemental Table S4) in the core indicated that the core collections are also an 357 excellent representative of phenotypic variations within the whole collection.

\section{Discussion}

\section{A-Genome Species Distribution, Einkorn Nomenclatures and Morphology}

362 Our results confirm that the WGRC A-genome collection includes arrays of naturally selected 363 germplasm around the center of origin (Figure 1). While verifying the morphologically based 364 grouping of A-genome species through population analysis, we identified three genetically different wild einkorn races (Figure 2 and 3). This information is very crucial in handling a large group of wild einkorn so that accessions with desired genetic background and morphology of

367 interest can be selected for utilization in breeding and further investigation. The wild einkorn genetic races described herein, matched with the races described in Kilian et al. (2007), add 
information to establish the evolutionary and genetic relationships between wild and

370 domesticated einkorn wheats. However, various nomenclature of the einkorn (Supplemental

371 Figure S11) creates a conundrum in interpreting the different races within the wild einkorn.

372 Some einkorn nomenclature is written in multiple languages; Schiemann (1948) published her

373 nomenclature in German and in Russian (Dorofeev et al., 1979), which could have reduced the

374 acceptance of the nomenclatures by the wider research communities. In a revised form of Mac

375 Key's classification (Mac Key, 2005b), the T. monococcum subsp. boeoticum was changed to $T$.

376 monococcum subsp. aegilopoides (Goncharov, 2011). Therefore, no single einkorn classification

377 is deemed to be the most widely accepted and uniformly used. The van Slageren (1994)

378 nomenclature that we follow also is mostly in agreement with the Mac Key classification,

379 because both systems use T. monococcum subsp. aegilopoides as the wild einkorn. With all

380 these issues, an updated and widely accepted monograph of einkorn may help maintaining

381 uniformity in taxonomy of these A-genome species.

383 Species and subspecies classification is first based on morphology. Multiple studies also have

384 discussed different ecogeographical wild einkorn races that have intermediate morphology. van

385 Zeist (1992) described two groups of wild einkorn: the first group (T. boeoticum subsp.

386 thaoudar) predominately exists in the southeast Turkey, northern Iraq, and west Iran, and the

387 second group (T. boeoticum subsp. aegilopoides) primarily occurs in the west Anatolian center

388 (van Zeist, 1992). The first group of accessions had a double-grained spikelet, and the second

389 group was single-grained, suggesting that the second group is more similar to domesticated

390 einkorn. (Brandolini and Heun, 2019) explained the T. boeoticum subsp. aegilopoides as an

391 intermediate type feral (semi-wild) einkorn with a semi-brittle rachis and T. boeoticum subsp.

392 thaoudar as the truly wild einkorn with an extremely brittle rachis and argued on the quantitative 393 nature of brittleness in einkorn wheat (Brandolini and Heun, 2019). They hypothesized that the

394 feral einkorn had evolved when agriculture moved from the southeast to western Turkey and the

395 Balkans. The semi-brittle rachis breaks into two parts only after being bent and the naturally

396 emerged semi-brittle rachis einkorn mutant still exists in the vicinity of the Karacadag, however,

397 the area is predominant for the truly wild double-grained einkorn (Brandolini and Heun, 2019).

398 Some einkorn accessions with intermediate leaf hairiness, a trait used to classify accessions that

399 is common only in wild einkorn, was also observed (Empilli et al., 2000), indicating that einkorn 
with intermediate or intergraded morphological characteristics are common (Harlan and Zohary,

401 1966). The three genetic races of wild einkorn observed in this study also possess unique genetic 402 relationships with cultivated einkorn, as shown by phylogenetic grouping and pairwise $\mathrm{F}_{\mathrm{ST}}$

403 values, showing the varying levels of relatedness within and between wild einkorn accession.

405 Genebank Curation

406 Globally, plant genebanks often suffer from identified and unidentified genetically identical 407 accessions that unnecessarily increase maintenance costs and more acutely can result in 408 duplicated efforts when characterizing and utilizing the germplasm (Díez et al., 2018). Here, we 409 curated 930 A-genome species accessions in WGRC genebank, identifying the identical and 410 misclassified accessions, and recognizing valuable unique accessions using genotyping. The 411 existence of misclassified accessions in the gene bank may be due to human error on class 412 assignment and/or data recording. It is also possible that some accessions might also have 413 controversial, intermediate or ambiguous morphology. Also, possible seed mixing during 414 germplasm management or germplasm exchanging between genebanks can lead confusion about 415 accessions, particularly as accessions are often renumbered when entered into a different 416 genebank and original matching accession numbers lost. As an example of misclassification, 417 consider the wild einkorn accession PI 427328 discussed earlier. Except for the WGRC and 418 Leibniz genebanks, three other collections have listed this accession (PI 427328) as T. urartu 419 (https://www.genesys-pgr.org/a/v2JRrMq2g22), illustrating the importance of genetic scrutiny of 420 the misclassified accessions within the A-genome accessions in different repositories.

422 One caveat is that the analysis was based on a single plant DNA and the variants were from a 423 single seed per accession. While the accessions were purified and derived from single plants, 424 inferences based on these data may not fully represent possible heterogeneity in various 425 accessions. Depending on the origin, maintenance, and propagation of different accessions in 426 different genebanks there may be varying levels of heterogeneity within a given accession. To 427 fully observe the heterogeneity of an accession, even for inbreeding species, testing multiple 428 individuals to represent a given accession is recommended. However, the observations we made 429 in the experiment even with a single plant genotyping provide an excellent picture of overall 430 diversity and roadmap for selecting optimal sets of accession for next step such as for breeding 
431 and core set development. While it is not advocated to remove accessions from the genebanks,

432 overall, this genotype-based curation can give needed focus to limited resources. Having a non-

433 redundant accession list, along with core collections, can reduce the genebank operation costs

434 and make germplasm preservation and utilization easier, particularly anytime the collection

435 needs to be increased or when phenotypic evaluations are undertaken.

\section{T. urartu: the Closest A-genome Diploid Relative of Wheat}

438 With GBS information here we showed that T. urartu is the closest diploid A-genome relative of 439 wheat and thereby most likely donor of A-genome to the hexaploid wheat (Supplemental Figure 440 S2). Most previous studies describing the relationship between T. urartu and wheat (Dvořák et 441 al., 1993) however relied on cytogenetic analysis. This study agrees with the known relationships 442 between wheat and A-genome diploids while using a much larger set of accession and molecular 443 markers $(\sim 1,000$ diploids and $>200$ wheat $)$ than previous studies.

\section{Wild Einkorn Races}

446 The wild einkorn groups were previously divided into $\alpha, \beta$, and $\gamma$ races (Kilian et al., 2007;

447 Zaharieva and Monneveux, 2014) which was consistent with the phylogeny observed in our 448 study. Furthermore, we validated these race groups to match accessions with common USDA PI 449 numbers in both studies overlapped their point of collection and almost all fall under the same 450 races in both studies (Supplemental Table S4). Comparing between studies, there were a few 451 discrepancies in race assignment of accessions (Kilian et al., 2007) that needed correction. For 452 example, Kilian et al. (2007) grouped PI 427328 in T. urartu, but our genetic analysis grouped it 453 into $\alpha$ race within subsp. aegilopoides which is also in harmony with WGRC database (accession 454 no. TA879). According to the Genesys database (https://www.genesys-

455 pgr.org/10.25642/IPK/GBIS/98704), another gene bank (Leibniz Institute of Plant Genetics and 456 Crop Plant Research) also classified this PI 427328 within wild einkorn but under the name $T$. 457 baeoticum Boiss. subsp. boeoticum exemplifying multiple wild einkorn nomenclatures use and 458 creating confusion when describing wild einkorn. Interestingly, Kilian et al. (2007) reported a 459 few feral types of einkorn accessions, indicating they are T. monococcum subsp. aegilopoides 460 according to the nomenclature used, which we did not observe in the WGRC collection. We 461 showed that the wild and domesticated einkorn can clearly be differentiated based on genomic 
462

463

464

465

\section{6}

467

468

469

470

471

472

473

474

475

476

477

478

479

480

481

482

483

484

485

486

487

488

489

490

491

492

data into $\alpha, \beta$, and $\gamma$ races and the domesticated accessions. Given the difficulty and ambiguity of morphological classification, the genetic classification from genomic data can be a preferred approach to cleanly classify any given accession.

\section{Population Analysis and Different Groups Under A-genome Species}

The population structure and $\mathrm{F}_{\mathrm{ST}}$ analysis on the A-genome species endorsed the established relationships between the species and subspecies. For instance, hybrids between T. monococcum and $T$. urartu are largely sterile and, hence, the genetic differentiation between these species is apparent (Fricano et al., 2014). Also, the intraspecific population differentiation between groups under einkorn at relatively higher $\mathrm{K}$ supported the known genetic relationship between these crossable subspecies that produce mostly fertile hybrids (Harlan and Zohary, 1966).

Our analysis shows that the $\alpha$ race einkorn accessions most likely represent the truly wild einkorn with an extremely brittle rachis, most likely the group of accessions that were traditionally classified as T. boeoticum subsp. thaoudar (Brandolini and Heun, 2019). Differentiation of subpopulations within the $\alpha$ race wild einkorn corresponding to geographic distribution implies migration and genetic drift among truly wild einkorn in the Near East. The $T$. urartu subgrouping of accessions from Lebanon and Turkey agrees with (Wang et al., 2017), where two subgroups, Mediterranean coastal and Mesopotamia-Transcaucasia, within T. urartu were reported (Wang et al., 2017).

\section{Diversity Analysis}

Cultivated einkorn had a lower Nei's diversity index (0.062) than the wild sister group and wild T. urartu (Table 1), which was expected. As a domesticated species, subsp. monococcum experienced a population bottleneck and artificial selection contributed to further limit genetic diversity. On the other hand, the population structure of cultivated einkorn did not show substantial admixture. With the exception of a few accessions, all individuals were true to single ancestry (Figure 2), suggesting a low post domestication admixture contributing elevated diversity. The involvement of a single race $(\beta)$ in domestication would have further reduced allelic diversity in the cultivated einkorn. Kilian et al. (2007) illustrated no nucleotide diversity was reduced during einkorn domestication; instead, they observed increased diversity in 
493 domesticated compared to wild einkorn (Kilian et al., 2007). However, the diversity assessment

494 in Kilian et al. (2007) could be influenced by the limited number of loci and smaller sample size;

495 especially, diversity estimates are sensitive to sample size when there are only a handful of

496 markers (Bashalkhanov et al., 2009; Li et al., 2009). In this experiment, we used thousands of

497 SNP markers and have larger number of accessions, which minimized the effect of sample size

498 and the number of loci. The highest Nei's diversity index (0.25) for all A-genome combined and

499 the considerably higher Nei's diversity index for each species and core collections indicated that

500 these accessions are very important assets with novel and useful genetic variations.

\section{Btr1: Einkorn Domestication Signal}

503 Through $\mathrm{F}_{\mathrm{ST}}$ computation, we showed that in einkorn wheat there is a single strong selection

504 signal observed on chromosome 3A corresponding to the Btr1 locus (Supplemental Figure S7).

505 Previous study also described Btrl as one of the most important features of einkorn

506 domestication (Pourkheirandish et al., 2018). The non-brittleness in domesticated einkorn is

507 controlled by a single nucleotide change in Btrl of wild einkorn that results in an amino acid

508 substitution (alanine to threonine) (Pourkheirandish et al., 2018). With 1,000 filtered loci per

509 chromosome, we located the candidate selection region. The availability of a T. monococcum

510 reference genome to call the genotype would be ideal for obtaining dense markers and better

511 locating the selection signature on einkorn wheat.

\section{Core Collections}

514 Establishing core collections of A-genome species enabled the harnessing of useful genetic

515 variation to improve wheat and cultivated einkorn; this genetic core of the A-genome species

516 included only 79 accessions and yet contains 98\% of the identified alleles while achieving a

517 more than 10-fold (79/930) reduction in the number of accessions (Table 1, Supplemental Table

518 S3). The higher Nei's diversity index computed for these core collections supported that they

519 have considerably higher relative diversity and can be leveraged for targeted germplasm

520 improvement.

521

\section{Conclusions}


523 This study reports the important aspects of the A-genome wheat species for genetic diversity, 524 gene bank curation, and core set selection. Following an assessment of nearly 1,000 accessions, 525 we report that the A-genome species possess a considerable amount of genetic diversity, which 526 can be utilized in breeding wheat and domesticated einkorn. This vast diversity is most

527 effectively managed in pre-breeding with well-defined core collections. Identifying and in-depth 528 characterizing of such core collections adds substantial value and accessibility to the germplasm. 529 Having a well curated and accurately described gene bank collection, as done here, is a critical 530 foundation to effectively using this rich diversity for crop improvement and enhancing the value 531 of gene bank resources.

\section{Materials and Methods}

Plant Resources

537 This study included 930 accessions of the A-genome diploid wheat species maintained in the 538 WGRC genebank (Supplemental Table S1), which were primarily acquired from the Near East, 539 Transcaucasia, and the Balkans (Figure 1). Most of the A-genome accessions ( 85\%) tested 540 include those initially collected by B. Lennert Johnson, University of California-Riverside in the 541 1960s and 1970s. The remaining accessions were obtained from gene banks in Japan (22), 542 Germany (24), and ICARDA (61). Several accessions were donated by Robert Metzger, USDA, 543 Oregon State University, Corvallis (26), seven were collected by the WGRC, and the remainder 544 (10) from other sources. We also tested 225 CIMMYT wheat lines (Supplemental Table S1) 545 genotyped earlier with GBS SNPs (Gao et al., 2021) thereby inferring the genetic relationships 546 between A-genome diploids and the hexaploid wheat.

\section{Genotypic Characterization}

549 The tested accessions were grown as single plants in the greenhouse and tissue collected in 96-

550 well plates. The tissues were lyophilized for $\sim 3$ days and ground to a fine powder using Retsch 551 mixer mill MM 400. Genomic DNA extraction and GBS library preparation steps were 552 according to (Singh et al., 2019). We had a total of four multiplexed GBS libraries including one 553 for the pilot study. The pilot study GBS library was 384-plex, whereas the other GBS libraries 
554 were 288-plex. We sequenced on the Illumina platform with 150 bp pair-end reads (PE150). The 555 information about GBS of 225 CIMMYT lines can be obtained (Gao et al., 2021).

557 The TASSEL5 GBSv2 pipeline was used for sequence data processing and genotype calling 558 (Glaubitz et al., 2014). Reads were aligned to a T. urartu pseudomolecule reference (Ling et al., 559 2018) using bowtie2 alignment (Langmead et al., 2009) and exported to variant call format 560 (VCF). Filtering of the VCF was done for bi-allelic SNPs using the Fisher exact test with a threshold p-value $<0.001$ as described previously (Poland et al., 2012b) considering that true variants should represent biallelic homozygous state for inbred accessions. Genotypes for accessions across all A-genome species were called together, followed by extracting variants 564 segregating within each species. The SNPs were filtered for minor allele frequency (MAF) > 5650.01 , missing $<30 \%$, and heterozygous $<10 \%$ at the population level unless otherwise stated in 566 the particular analysis method below (e.g., different filtration was used for allele matching and 567 identifying identical accessions) (Supplemental Figure S12). The percentage missing per genotype was computed and removed any genotype with missing $>55 \%$. The imputation was done only once in a separated VCF file for T. monococcum (einkorn) group which was used to compute genome-wise $\mathrm{F}_{\mathrm{ST}}$ statistics for selection signatures (Supplemental Figure S12). The R codes used to process the hapmap file and downstream analysis and all required data files are provided as supplemental documents at Dryad digital repository (doi:10.5061/dryad.9zw3r22f6).

574 The A-genome diploids and wheat lines were genotyped together calling variants on the Agenome of wheat reference genome of Chinese Spring (iwgsc_refseqv1.0) (Appels et al., 2018).

576 We also filtered these SNPs using aforementioned criteria. The unrooted neighbor-joining (NJ)

577 phylogenetic tree of A-genome diploid and wheat lines were generated for investigating the genetic relationship. We followed approach of (Singh et al., 2019) to generate NJ tree from GBS

579 sampled population, where clustering was conducted with default parameters of R packages 580 'dist', 'ape', and 'phyclust'.

\section{Gene Bank Curation}

583 A-genome species in the WGRC gene bank were curated to identify misclassified and 584 genetically identical accessions. The misclassified accessions identified based on the genetic 
properties were compared with accessions in the adjusted class morphologically to assure if they 586 were previously assigned or documented to the wrong class. Furthermore, to confirm the ploidy 587 of the misclassified accessions that were grouped far from the major T. urartu clade and did not 588 exhibit a closer relationship with any diploid A-genome in genetic tree, chromosome counts were 589 made by staining with 4',6-diamidino-2-phenylindole (DAPI) following the details and methods of (Koo et al., 2017).

592 The genetically identical accessions were identified using pairwise allele matching across 593 homozygous and non-missing sites. We first analyzed the loci identity proportions distribution at 594 genome-wide scale including every possible pair-wise comparison among accessions within a 595 single species. A threshold for allele matching percentage given discrepancies for sequencing errors was then detected by finding a point that separates the local maxima existing around the prefect identity (100\%). The identity matrix and percentage allele matching were computed in $\mathrm{R}$ using a custom script as described by (Singh et al., 2019). The morphological similarity and the geographical relations of the genetically identical accessions were checked for confirmation.

600 Glume color (level of darkness) was used as a morphological marker for cross-validation to 601 affirm the accessions in a genetically identical set have the same or similar phenotypes. The 602 variation in glume color was rated from completely white (0) to dark black (9).

\section{Population Structure}

605 Population structure of A-genome wheat species was analyzed using fastStructure (Raj et al., 2014). The filtered genotyping file (Table 1) for the entire population was used to generate input

607 data file for the fastStructure extracting only sites with segregating homozygous loci, where the 608 het genotype calls were converted to missing (doi:10.5061/dryad.9zw3r22f6). The fastStructure 609 was initially run at $\mathrm{K}=2$ to $\mathrm{K}=12$ with three replications using 'simple' prior where $\mathrm{K}$ refers to 610 number of population or model complexity. The value of $\mathrm{K}$ was considered optimum if no 611 further distinct differentiations occurred in the population (Supplemental Figure S13 and S14).

612 An appropriate number of $\mathrm{K}$ was also obtained using the fastStructure provided utility tool,

613 chooseK.py. For the optimum number of $\mathrm{K}$, the program was run using 'logistic' prior at $\mathrm{K}=2$ to $614 \mathrm{~K}=7$ with three replications (Singh et al., 2019). The fastStructure output was graphically 615 visualized using an R package POPHELPER (Francis, 2017). Passport information including the 
616 classification based on morphology, and the accessions geographical sites were used to group

617 and reorder the samples in population analysis. Accessions that were identified as misclassified

618 were confirmed through morphological evaluation and reordered to subspecies based on the

619 genotype-based grouping and the curated results were plotted.

621 Phylogenetic clustering was carried out in R using 'dist' function

622 (https://www.rdocumentation.org/packages/amap/versions/0.8-18/topics/Dist) and 'ape' (Paradis 623 and Schliep, 2019) and 'phyclust' (Chen, 2011) packages as in (Singh et al., 2019). The branches

624 of an unrooted neighbor-joining (NJ) tree were first colored using the morphology-based

625 classification, and then according to genotype analysis. The morphology-based coloring was

626 particularly focused in identifying misclassified accessions. A-genome species population

627 genetic structure was also dissected using principal component analysis (PCA) of genomic data.

628 For PCA, we estimated the eigenvalues and eigenvectors on R using the 'e' function in A matrix 629 obtained from the rrBLUP (Endelman, 2011; Singh et al., 2019).

\section{Analysis of Genetic Diversity}

632 A-genome species genetic diversity was assessed by computing the Nei's diversity index (Nei,

633 1973) using filtered genotyping markers. We computed the Nei's indices of (1) all A-genome

634 accessions together, (2) each species and subspecies independently, (3) the races within the

635 subspecies, and (4) and the core collections. Number of segregating loci per group were

636 determined (Table 1). The minor allele frequency (MAF) for each species was also plotted to

637 discern the proportions of rare variants in respective population. A pairwise fixation index $\left(\mathrm{F}_{\mathrm{ST}}\right)$

638 (Nei, 1987) also was computed between the species and subgroups separated by the population

639 analysis (Singh et al., 2019).

\section{$641 \quad$ F $_{\text {ST }}$ Within Einkorn and Selection signature}

642 We computed a genome-wide $\mathrm{F}_{\mathrm{ST}}$ statistic for variants within the einkorn group using $\mathrm{R}$ ( $\mathrm{R}$ Core

643 Team 2019) as described (Porto-Neto et al., 2013). This method computes $F_{\text {ST }}$ statistic based on

644 pure drift model (Nicholson et al., 2002). We also compared the output by computing the

645 Cockerham and Weir $F_{\text {ST }}$ statistic (Weir and Cockerham, 1984) using VCFtools (Danecek et al.,

646 2011). The T. monococcum VCF file separated from the original VCF with biallelic variants was 
647 further filtered keeping SNPs with MAF > 0.01, missing < 30\% and heterozygous $<10 \%$

648 followed by imputation using Beagle 5.1 (Browning et al., 2018). The filtered and imputed

649 genotyping information was used to derive the $\mathrm{F}_{\mathrm{ST}}$ values. To balance the population sizes of

650 domesticated and wild einkorn, we randomly chose 145 wild einkorn accessions to match the

651 number of 145 domesticated accessions. The $\mathrm{F}_{\mathrm{ST}}$ were plotted using ggplot 2 in $\mathrm{R}$ (R Core Team

652 2019) and the raw $F_{\text {ST }}$ plots were smoothed using Lowess method (Pintus et al., 2014) to find the

653 genomic regions with extreme $\mathrm{F}_{\mathrm{ST}}$. To define the selection signal peak, we considered outlier $\mathrm{F}_{\mathrm{ST}}$

654 values that were more than three standard deviation $(3 \sigma)$ over genome wide average as the

655 threshold.

656

\section{Core Collections}

658 Core collections of T. urartu, and T. monococcum (wild and domesticated einkorn) were selected

659 taking allelic diversity, genotype coverage, geographical representation, and phenotypic variation

660 (glume color) into consideration. From the genotyping file containing segregated loci (Table 1),

661 heterozygous genotypes were masked before running the core accessions selection software

662 GenoCore (Jeong et al., 2017). We ran GenoCore with the default parameters: -d 0.01\% and -cv

$66399 \%$. The positions of the selected samples within the phylogenetic tree and PCA clusters were

664 observed through coloring the selected core accessions versus all other samples. Also, the

665 geographical representations were evaluated marking the selected vs. remaining accessions in the

666 google map using GPS Visualizer (https://www.gpsvisualizer.com). To ensure phenotypic

667 variations in the selected core sets, we considered the glume color score (Supplemental Table

668 S4) as a reference variation. The Nei's diversity index (1987) of core sets were also computed

669 (Nei, 1987).

670 


\section{Accession Numbers}

674 Raw sequence data obtained from GBS, the fastq files, has been deposited at the National Center

675 for Biotechnology Information (NCBI) SRA database with the BioProject accession

676 PRJNA744683 (https://www.ncbi.nlm.nih.gov/sra/PRJNA744683). The GBS key file with

677 required information for demultiplexing and further detail about the SRA deposited fastq files

678 can be obtained at Dryad digital repository (doi:10.5061/dryad.9zw3r22f6).

680 All data are available in the manuscript or the supplemental resources and at the Dryad digital 681 repositories https://datadryad.org/stash/dataset/doi:10.5061\%2Fdryad.9zw3r22f6

\section{Supplemental Data}

684 Supplemental Figure S1. The T. urartu clade and subsp. aegilopoides $\alpha$ race clade in a

685 unrooted Neighbor-Joining (NJ) tree.

Supplemental Figure S2. Threshold determination for declaring identifying genetically identical 688 accessions.

Supplemental Figure S3. An unrooted Neighbor-Joining (NJ) tree of wheat (T. aestivum L.) and A-genome species: T. urartu, subsp. aegilopoides, and subsp. monococcum.

693 Supplemental Figure S4. Chromosome count for ploidy level of a putative misclassified wild 694 wheat accession TA10881

Supplemental Figure S5. Principle component analysis (PCA) plot for A-genome wheat species with two major PCs.

701 Supplemental Figure S7. The fixation index $\left(\mathrm{F}_{\mathrm{ST}}\right)$ curve showing selection signal for einkorn 702 wheat on chromosome 3A. 
704 Supplemental Figure S8. An unrooted Neighbor-Joining (NJ) phylogenetic tree of A-genome

705 wheat species showing the accessions in the core collections and all other accessions in

706 respective clades.

708 Supplemental Figure S9. Principle component analysis (PCA) plot of A-genome wheat

709 accessions indicating all versus core accessions.

711 Supplemental Figure S10. Geographic map of A-genome diploid wheat accessions (small

712 markers) and the accessions in core collection (large markers).

714 Supplemental Figure S11. Diagram showing three different taxonomic classification systems of 715 einkorn wheat.

717 Supplemental Figure S12. Flow diagram showing filtering criteria for different subsets of A-

718 genome diploid collection used to generate single nucleotide polymorphism (SNP) matrixes.

720 Supplemental Figure S13. Determining optimum value of K for fastStructure analysis of A721 genome species.

723 Supplemental Figure S14. Admixture analysis from $\mathrm{K}=2$ to $\mathrm{K}=12$ with corresponding regions 724 of collection noted for each group.

726 Supplemental Table S1. List of A-genome accessions, their origin, and the genetically identical 727 set (separate file).

729 Supplemental Table S2. The misclassified A-genome species accessions, their previous class based on morphology, and the updated class/group based on the genotyping. 
733 Supplemental Table S3. Number of accessions with common PI numbers that clustered in

734 corresponding groups in this experiment and a past experiment.

735

736 Supplemental Table S4. Core collections of A-genome species (separate file).

738 Supplemental Table S5. Pairwise fixation index $\left(\mathrm{F}_{\mathrm{ST}}\right)$ coefficients among the subgroups within $739 \alpha$ race of subsp. aegilopoides (wild einkorn) and the admixture groups.

741 Supplemental Text S1. Coding sequence of gene for non-brittle rachis 1 (Btrl) in T.

742 monococcum subsp. monococcum.

\section{Funding information}

745 This material is based upon work supported by the U.S. National Science Foundation and

746 Industry Partners under Award No. (1822162) "Phase II IUCRC at Kansas State University

747 Center for Wheat Genetic Resources" and the National Science Foundation under Grant No.

748 (1339389) “GPF-PG: Genome Structure and Diversity of Wheat and Its Wild Relatives”. Any

749 opinions, findings, and conclusions or recommendations expressed in this material are those of

750 the author(s) and do not necessarily reflect the views of the National Science Foundation or

751 industry partners.

\section{Acknowledgements}

754 We would like to thank the Wheat Genetic Resource Center (WGRC) gene bank for collecting 755 and maintaining these A-genome species.

757 Authors have no competing interests 
Tables

761 Table 1. A-genome species and sub-species groups with number of samples, the Nei's diversity

762 indices, and number of segregating loci. The percentage of segregating SNPs for core set groups

763 were estimated relative to the segregating loci within the respective groups.

764

\begin{tabular}{|c|c|c|c|}
\hline Group & $\begin{array}{l}\text { Number of } \\
\text { Samples }\end{array}$ & $\begin{array}{l}\text { Diversity } \\
\text { Index }\end{array}$ & $\begin{array}{l}\text { Segregating } \\
\text { SNPs }\end{array}$ \\
\hline $\begin{array}{l}\text { A-genome species }(T . \text { monococcum }+T \text {. } \\
\text { urartu) }\end{array}$ & 925 & 0.25 & 13860 \\
\hline T. monococcum (einkorn) & 729 & 0.106 & $8989(64.8 \%)$ \\
\hline Domesticated einkorn (subsp. & 145 & 0.062 & $4401(31.7 \%)$ \\
\hline monococcum) & & & \\
\hline Wild einkorn (subsp. aegilopoides) & 584 & 0.087 & $8283(59.7 \%)$ \\
\hline$\alpha$ race einkorn & 524 & 0.075 & $7159(51.6 \%)$ \\
\hline$\gamma$ race einkorn & 48 & 0.095 & $5422(39.2 \%)$ \\
\hline$\beta$ race einkorn & 12 & 0.065 & $3402(24.5 \%)$ \\
\hline T. urartu & 196 & 0.066 & $3840(27.7 \%)$ \\
\hline A-genome species core set & 79 & 0.261 & $13654(98.5 \%)$ \\
\hline T. monococcum core & 60 & 0.117 & $7416(82.5 \%)$ \\
\hline Domesticated einkorn core & 19 & 0.068 & $3765(85.5 \%)$ \\
\hline Wild einkorn core & 41 & 0.099 & $6576(79.4 \%)$ \\
\hline T. urartu core & 19 & 0.066 & $3286(85.5 \%)$ \\
\hline
\end{tabular}

765

766 
767 Table 2. Number (\#) of unique accessions, number of accessions in a set consisting maximum 768 identical accessions, and total accessions of A-genome species: T. urartu, domesticated einkorn 769 (subsp. monococcum), and wild einkorn (subsp. aegilopoides) three genetic races: $\alpha, \gamma$, and $\beta$. 770 The identical accessions were detected using pairwise allele matching. 771

\begin{tabular}{llllll}
\hline & $\alpha$ race & $\gamma$ race & $\beta$ race & subsp. monococcum & T. urartu \\
\cline { 2 - 6 } Total accessions & $\mathbf{5 2 4}$ & $\mathbf{4 8}$ & $\mathbf{1 2}$ & $\mathbf{1 4 5}$ & $\mathbf{1 9 6}$ \\
\# Loci compared & 4112 & 4112 & 4112 & 3337 & 6356 \\
Identical $^{\psi}$ & 28 & 3 & 0 & 5 & 39 \\
Unique accessions & $\mathbf{1 9 8}(\mathbf{3 7 . 8 \% )}$ & $\mathbf{3 7 ( 7 7 \% )}$ & $\mathbf{1 2}(\mathbf{1 0 0 \% )}$ & $\mathbf{9 7}(\mathbf{6 6 . 8 \%})$ & $\mathbf{6 1}$ \\
& & & & & $\mathbf{( 3 1 . 2 \% )}$
\end{tabular}

772

$773 \psi=$ Number of accessions in a set that represent the largest group of genetically identical

774 accessions

775 
776 Table 3. Pairwise $F_{S T}$ coefficients among the A-genome wheat species. Higher $\mathrm{F}_{\mathrm{ST}}$ reflects a

777 stronger population differentiation. The $\alpha, \beta$ and $\gamma$ genetic races comprise the wild einkorn ( $T$.

778 monococcum subsp. aegilopoides L.).

779

\begin{tabular}{lllll}
\hline & $\alpha$ race & $\gamma$ race & $\beta$ race & T. urartu \\
\hline subsp. monococcum & 0.56 & 0.41 & 0.31 & 0.87 \\
$\alpha$ race & - & 0.40 & 0.50 & 0.86 \\
$\gamma$ race & - & - & 0.37 & 0.83 \\
$\beta$ race & - & - & - & 0.86 \\
\hline
\end{tabular}

780

781 
784 Figure 1. Geographic distribution of A-genome wheat species maintained in the Wheat Genetics

785 Resource Center (WGRC) gene bank. Collection sites of accessions in this study are designated

786 for domesticated einkorn (Triticum. monococcum subsp. monococcum) (blue); $\alpha$ race within wild

787 einkorn (T. monococcum. subsp. aegilopoides) (red); $\gamma$ race wild einkorn (orange); $\beta$ race wild

788 einkorn (magenta); and T. urartu (yellow).

Figure 2. Population structure of A-genome wheat species (Triticum monococcum L. and T. urartu), where description of the groups and colors refers to $K=7$. Subpopulations were determined using fastStructure. Each color represents a population, and each bar indicates the admixture proportion of an individual accession from K populations. The subgroup within $\alpha$, which is exemplified by orange color includes the accessions from Erbil (also spelled Arbil), Iraq, whereas the subgroup embodied by red yellow only comprises the accessions from Duhok (ancient name 'Dahuk', Iraq). The bars with red color only represent the accessions from southeast Turkey (ST). Other admixture types within $\alpha$ included accessions were from Iran, SU (Sulaymaniyah (Iraq)), random different sites (D) and unknown sites (U) as indicated. Within $T$. urartu, the LE group represents accessions from Lebanon, the TU includes accession from Turkey, S indicates accessions from Syria and M shows accessions from mixed sites.

Figure 3. An unrooted Neighbor-Joining (NJ) tree of A-genome species: T. urartu, subsp. aegilopoides, and subsp. monococcum. The tree branches are colored based on the genetic

804 grouping of the accessions after correcting misclassified accessions. T. urartu (yellow), 805 domesticated einkorn (blue), wild einkorn race $\alpha$ (red), wild einkorn race $\gamma$ (orange), and wild einkorn race $\beta$ (magenta) are shown.

Figure 4. Relationship between the allele coverage as estimated using GenoCore and the number of samples selected in the core for einkorn group (T. monococcum). The threshold for 60 accessions at approximately $90 \%$ genotype coverage is shown with vertical red line. 
Appels, R., Eversole, K., Feuillet, C., et al. 2018. Shifting the limits in wheat research and breeding using a fully annotated reference genome. Science, 361.

Bashalkhanov, S., Pandey, M. \& Rajora, O. P. 2009. A simple method for estimating genetic diversity in large populations from finite sample sizes. BMC Genetics, 10, 84.

Brandolini, A. \& Heun, M. 2019. Genetics of brittleness in wild, domesticated and feral einkorn wheat (Triticum monococcum L.) and the place of origin of feral einkorn. Genetic Resources and Crop Evolution, 66, 429-439.

Brandolini, A., Volante, A. \& Heun, M. 2016. Geographic differentiation of domesticated einkorn wheat and possible Neolithic migration routes. Heredity, 117, 135-141.

Browning, B. L., Zhou, Y. \& Browning, S. R. 2018. A One-Penny Imputed Genome from NextGeneration Reference Panels. The American Journal of Human Genetics, 103, 338-348.

Brunazzi, A., Scaglione, D., Talini, R. F., et al. 2018. Molecular diversity and landscape genomics of the crop wild relative Triticum urartu across the Fertile Crescent. The Plant Journal, 94, 670-684.

Charmet, G. 2011. Wheat domestication: Lessons for the future. Comptes Rendus Biologies, 334, 212-220.

Chen, S., Guo, Y., Briggs, J., et al. 2018. Mapping and characterization of wheat stem rust resistance genes SrTm5 and Sr60 from Triticum monococcum. Theoretical and Applied Genetics, 131, 625-635.

Chen, W.-C. 2011. Overlapping codon model, phylogenetic clustering, and alternative partial expectation conditional maximization algorithm. Iowa State University.

Comai, L. 2005. The advantages and disadvantages of being polyploid. Nature Reviews Genetics, 6, 836-846.

Danecek, P., Auton, A., Abecasis, G., et al. 2011. The variant call format and VCFtools. Bioinformatics, 27, 2156-2158.

Díez, M. J., De La Rosa, L., Martín, I., et al. 2018. Plant Genebanks: Present Situation and Proposals for Their Improvement. the Case of the Spanish Network. Frontiers in Plant Science, 9.

Dong, L., Huo, N., Wang, Y., et al. 2012. Exploring the diploid wheat ancestral A genome through sequence comparison at the high-molecular-weight glutenin locus region. Molecular Genetics and Genomics, 287, 855-866.

Dorofeev, V., Filatenko, A., Migušova, E., et al. 1979. Pšenica (Wheat). Kolos”, Leningrad.

Dvorak, J., Mcguire, P. E. \& Cassidy, B. 1988. Apparent sources of the A genomes of wheats inferred from polymorphism in abundance and restriction fragment length of repeated nucleotide sequences. Genome, 30, 680-689.

Dvořák, J., Terlizzi, P. D., Zhang, H.-B., et al. 1993. The evolution of polyploid wheats: identification of the A genome donor species. Genome, 36, 21-31.

Empilli, S., Castagna, R. \& Brandolini, A. 2000. Morpho-agronomic variability of the diploid wheat Triticum monococcum L. Plant Genetic Resources Newsletter, 36-40.

Endelman, J. B. 2011. Ridge Regression and Other Kernels for Genomic Selection with R Package rrBLUP. The Plant Genome, 4, 250-255.

Feldman, M. \& Levy, A. A. 2012. Genome evolution due to allopolyploidization in wheat. Genetics, 192, 763-774. 
Francis, R. M. 2017. pophelper: an R package and web app to analyse and visualize population structure. Molecular Ecology Resources, 17, 27-32.

Fricano, A., Brandolini, A., Rossini, L., et al. 2014. Crossability of Triticum urartu and Triticum monococcum wheats, homoeologous recombination, and description of a panel of interspecific introgression lines. G3 (Bethesda, Md.), 4, 1931-1941.

Gao, L., Koo, D.-H., Juliana, P., et al. 2021. The Aegilops ventricosa 2NvS segment in bread wheat: cytology, genomics and breeding. Theoretical and Applied Genetics, 134, 529542.

Glaubitz, J. C., Casstevens, T. M., Lu, F., et al. 2014. TASSEL-GBS: A High Capacity Genotyping by Sequencing Analysis Pipeline. PLOS ONE, 9, e90346.

Goncharov, N. P. 2011. Genus Triticum L. taxonomy: the present and the future. Plant Systematics and Evolution, 295, 1-11.

Harlan, J. R. \& Zohary, D. 1966. Distribution of Wild Wheats and Barley. Science, 153, 10741080.

Heun, M., Schäfer-Pregl, R., Klawan, D., et al. 1997. Site of Einkorn Wheat Domestication Identified by DNA Fingerprinting. Science, 278, 1312-1314.

Jeong, S., Kim, J.-Y., Jeong, S.-C., et al. 2017. GenoCore: A simple and fast algorithm for core subset selection from large genotype datasets. PLOS ONE, 12, e0181420.

Kilian, B., Martin, W. \& Salamini, F. 2010. Genetic Diversity, Evolution and Domestication of Wheat and Barley in the Fertile Crescent. In: GLAUBRECHT, M. (ed.) Evolution in Action: Case studies in Adaptive Radiation, Speciation and the Origin of Biodiversity. Berlin, Heidelberg: Springer Berlin Heidelberg.

Kilian, B., Özkan, H., Walther, A., et al. 2007. Molecular Diversity at 18 Loci in 321 Wild and 92 Domesticate Lines Reveal No Reduction of Nucleotide Diversity during Triticum monococcum (Einkorn) Domestication: Implications for the Origin of Agriculture. Molecular Biology and Evolution, 24, 2657-2668.

Kolmer, J. A., Anderson, J. A. \& Flor, J. M. 2010. Chromosome Location, Linkage with Simple Sequence Repeat Markers, and Leaf Rust Resistance Conditioned by Gene Lr63 in Wheat. Crop Science, 50, 2392-2395.

Koo, D.-H., Liu, W., Friebe, B., et al. 2017. Homoeologous recombination in the presence of $\mathrm{Ph} 1$ gene in wheat. Chromosoma, 126, 531-540.

Langmead, B., Trapnell, C., Pop, M., et al. 2009. Ultrafast and memory-efficient alignment of short DNA sequences to the human genome. Genome biology, 10, 1-10.

Li, O., Zhao, Y.-Y., Guo, N., et al. 2009. Effects of sample size and loci number on genetic diversity in wild population of grass carp revealed by SSR.

Ling, H.-Q., Ma, B., Shi, X., et al. 2018. Genome sequence of the progenitor of wheat A subgenome Triticum urartu. Nature, 557, 424-428.

Mac Key, J. 1954. The taxonomy of hexaploid wheat. Svensk Bot Tidskr, 48, 579-590.

Mac Key, J. 2005a. Wheat: its concept, evolution and taxonomy. Durum wheat breeding. Current approaches and future strategies, 1, 3-61.

Mac Key, J. 2005b. Wheat: its concept, evolution, and taxonomy. Durum Wheat Breeding. CRC Press.

Middleton, C. P., Senerchia, N., Stein, N., et al. 2014. Sequencing of chloroplast genomes from wheat, barley, rye and their relatives provides a detailed insight into the evolution of the Triticeae tribe. PloS one, 9, e85761-e85761. 
904

905

906

907

908

909

910

911

912

913

914

915

916

917

918

919

920

921

922

923

924

925

926

927

928

929

930

931

932

933

934

935

936

937

938

939

940

941

942

943

944

945

946

947

948

949

Mondal, S., Rutkoski, J. E., Velu, G., et al. 2016. Harnessing Diversity in Wheat to Enhance Grain Yield, Climate Resilience, Disease and Insect Pest Resistance and Nutrition Through Conventional and Modern Breeding Approaches. Frontiers in Plant Science, 7.

Nair, K. P. 2019. Utilizing crop wild relatives to combat global warming. Advances in Agronomy. Elsevier.

Nei, M. 1973. Analysis of Gene Diversity in Subdivided Populations. Proceedings of the National Academy of Sciences, 70, 3321-3323.

Nei, M. 1987. Molecular Evolutionary Genetics Columbia University Press New York 512.

Nicholson, G., Smith, A. V., Jónsson, F., et al. 2002. Assessing population differentiation and isolation from single-nucleotide polymorphism data. Journal of the Royal Statistical Society: Series B (Statistical Methodology), 64, 695-715.

Özkan, H., Tuna, M., Kilian, B., et al. 2010. Genome size variation in diploid and tetraploid wild wheats. AoB PLANTS, 2010.

Paradis, E. \& Schliep, K. 2019. ape 5.0: an environment for modern phylogenetics and evolutionary analyses in R. Bioinformatics, 35, 526-528.

Pintus, E., Sorbolini, S., Albera, A., et al. 2014. Use of locally weighted scatterplot smoothing (LOWESS) regression to study selection signatures in Piedmontese and Italian Brown cattle breeds. Anim Genet, 45, 1-11.

Poland, J., Endelman, J., Dawson, J., et al. 2012b. Genomic selection in wheat breeding using genotyping-by-sequencing. The Plant Genome, 5.

Poland, J. A., Brown, P. J., Sorrells, M. E., et al. 2012a. Development of high-density genetic maps for barley and wheat using a novel two-enzyme genotyping-by-sequencing approach. PloS one, 7, e32253-e32253.

Porto-Neto, L. R., Lee, S. H., Lee, H. K., et al. 2013. Detection of Signatures of Selection Using FST. In: GONDRO, C., VAN DER WERF, J. \& HAYES, B. (eds.) Genome-Wide Association Studies and Genomic Prediction. Totowa, NJ: Humana Press.

Pourkheirandish, M., Dai, F., Sakuma, S., et al. 2018. On the Origin of the Non-brittle Rachis Trait of Domesticated Einkorn Wheat. Frontiers in Plant Science, 8.

Raj, A., Stephens, M. \& Pritchard, J. K. 2014. fastSTRUCTURE: Variational Inference of Population Structure in Large SNP Data Sets. Genetics, 197, 573-589.

Rouse, M. N. \& Jin, Y. 2011. Stem Rust Resistance in A-Genome Diploid Relatives of Wheat. Plant Disease, 95, 941-944.

Saintenac, C., Zhang, W., Salcedo, A., et al. 2013. Identification of Wheat Gene <em>Sr35</em> That Confers Resistance to Ug99 Stem Rust Race Group. Science, 341, 783-786.

Schiemann, E. 1948. Weizen. Roggen, Gerste: Systematik Geschichte und Verwendung, Verlag Gustav Fischer, Jena, Germany.

Sheedy, J. G., Thompson, J. P. \& Kelly, A. 2012. Diploid and tetraploid progenitors of wheat are valuable sources of resistance to the root lesion nematode Pratylenchusthornei. Euphytica, 186, 377-391.

Singh, N., Wu, S., Tiwari, V., et al. 2019. Genomic Analysis Confirms Population Structure and Identifies Inter-Lineage Hybrids in Aegilops tauschii. Frontiers in Plant Science, 10.

Van Slageren, M. W. 1994. Wild wheats: a monograph of Aegilops L. and Amblyopyrum (Jaub. \& Spach) Eig (Poaceae). Wageningen Agricultural University Papers.

Van Zeist, W. 1992. The origin and development of plant cultivation in the Near East. Nichibunken Japan Review, 149-165. 
950 Wang, X., Luo, G., Yang, W., et al. 2017. Genetic diversity, population structure and markertrait associations for agronomic and grain traits in wild diploid wheat Triticum urartu. BMC Plant Biology, 17, 112.

953 Weir, B. S. \& Cockerham, C. C. 1984. Estimating F-statistics for the analysis of population structure. evolution, 1358-1370.

Wulff, B. B. H. \& Moscou, M. J. 2014. Strategies for transferring resistance into wheat: from wide crosses to GM cassettes. Frontiers in Plant Science, 5.

Zaharieva, M. \& Monneveux, P. 2014. Cultivated einkorn wheat (Triticum monococcum L. subsp. monococcum): the long life of a founder crop of agriculture. Genetic Resources and Crop Evolution, 61, 677-706. 


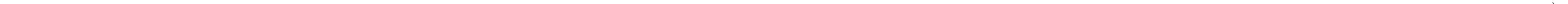




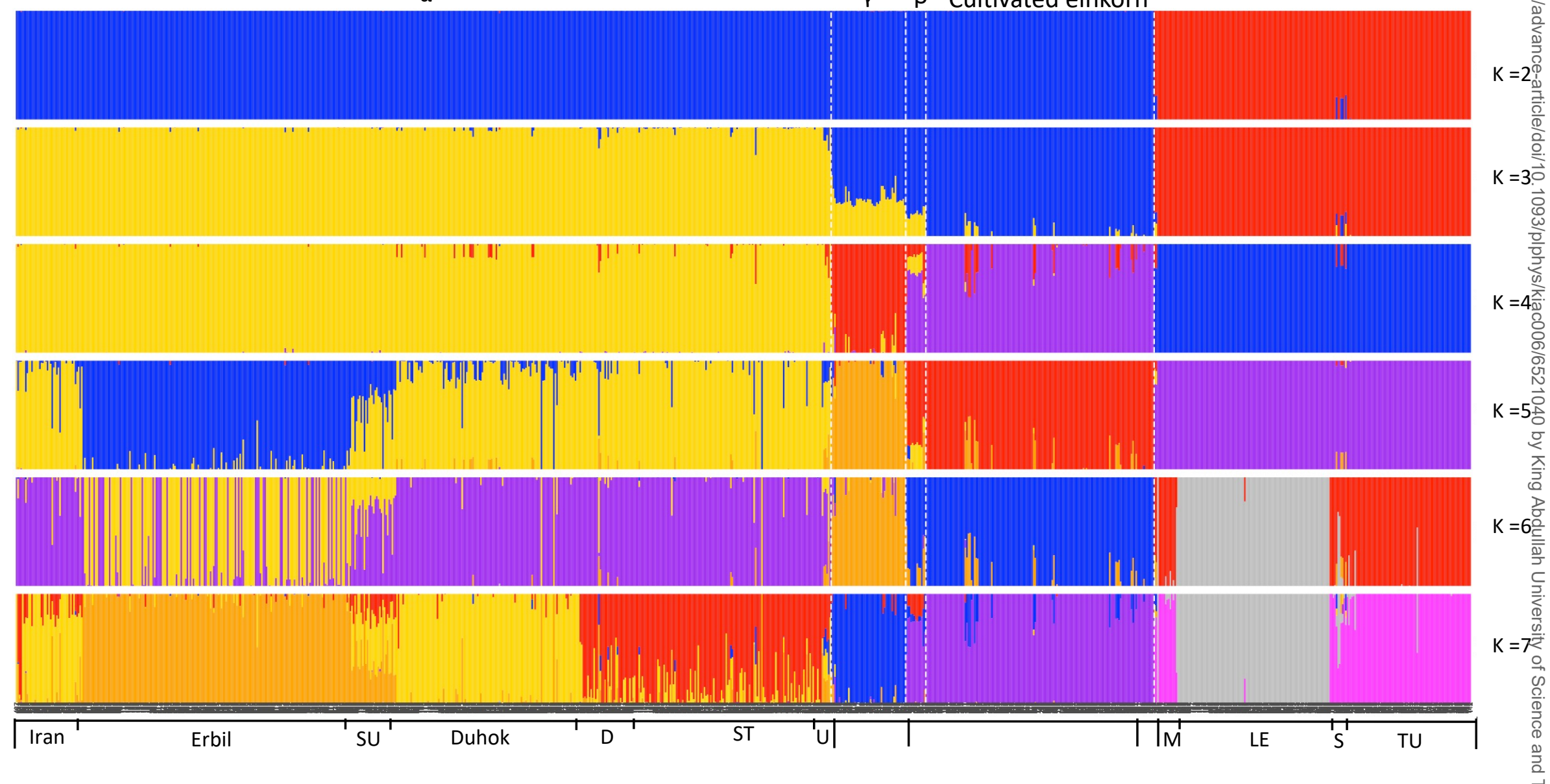




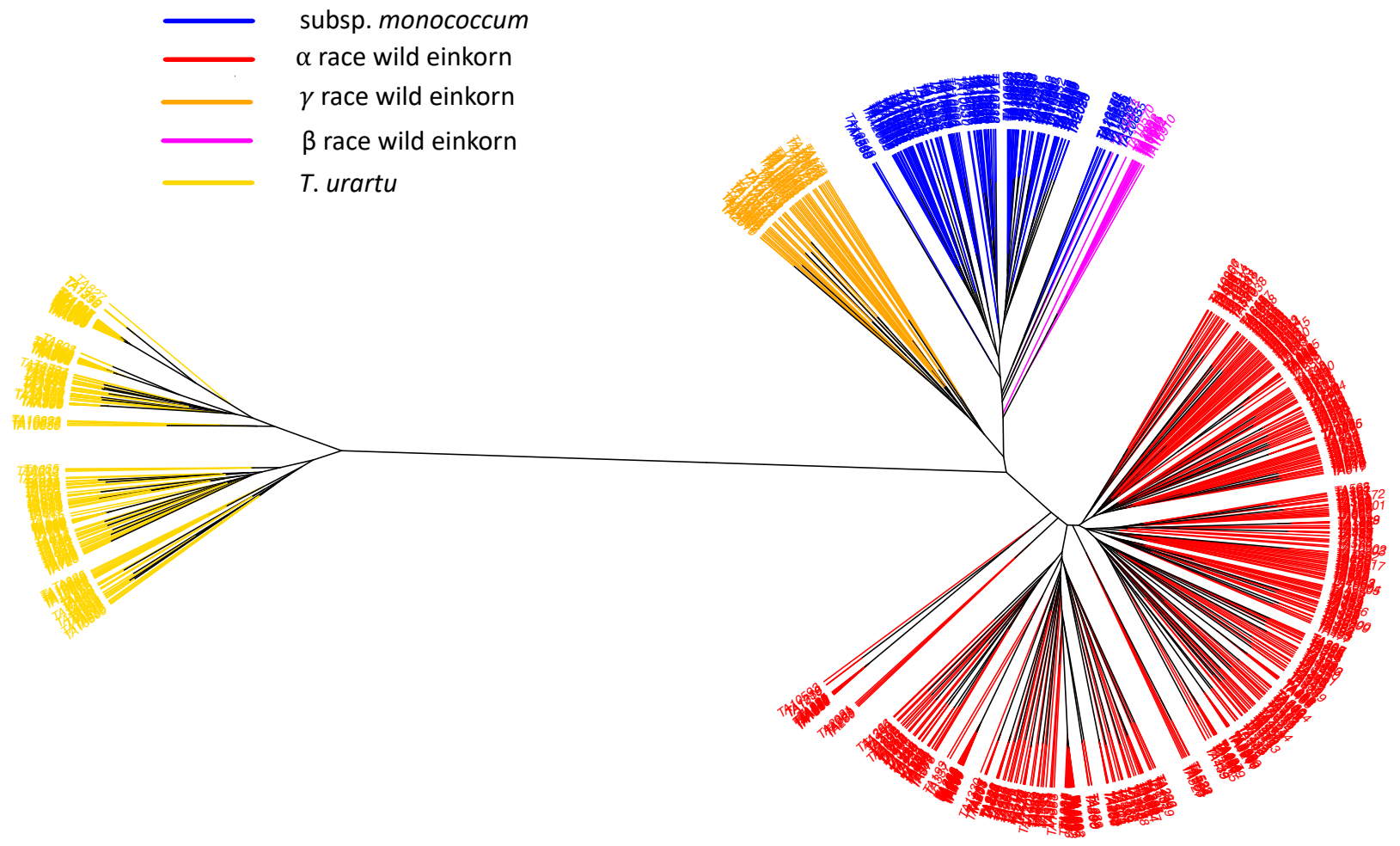

Figure 3. An unrooted Neighbor-Joining (NJ) tree of A-genome species: T. urartu, subsp. aegilopoides, and subsp. monococcum. The tree branches are colored based on the genetic grouping of the accessions after correcting misclassified accessions. T. urartu (yellow), domesticated einkorn (red), wild einkorn race $\alpha$ (blue), and wild einkorn race $\gamma$ (green) are shown. 


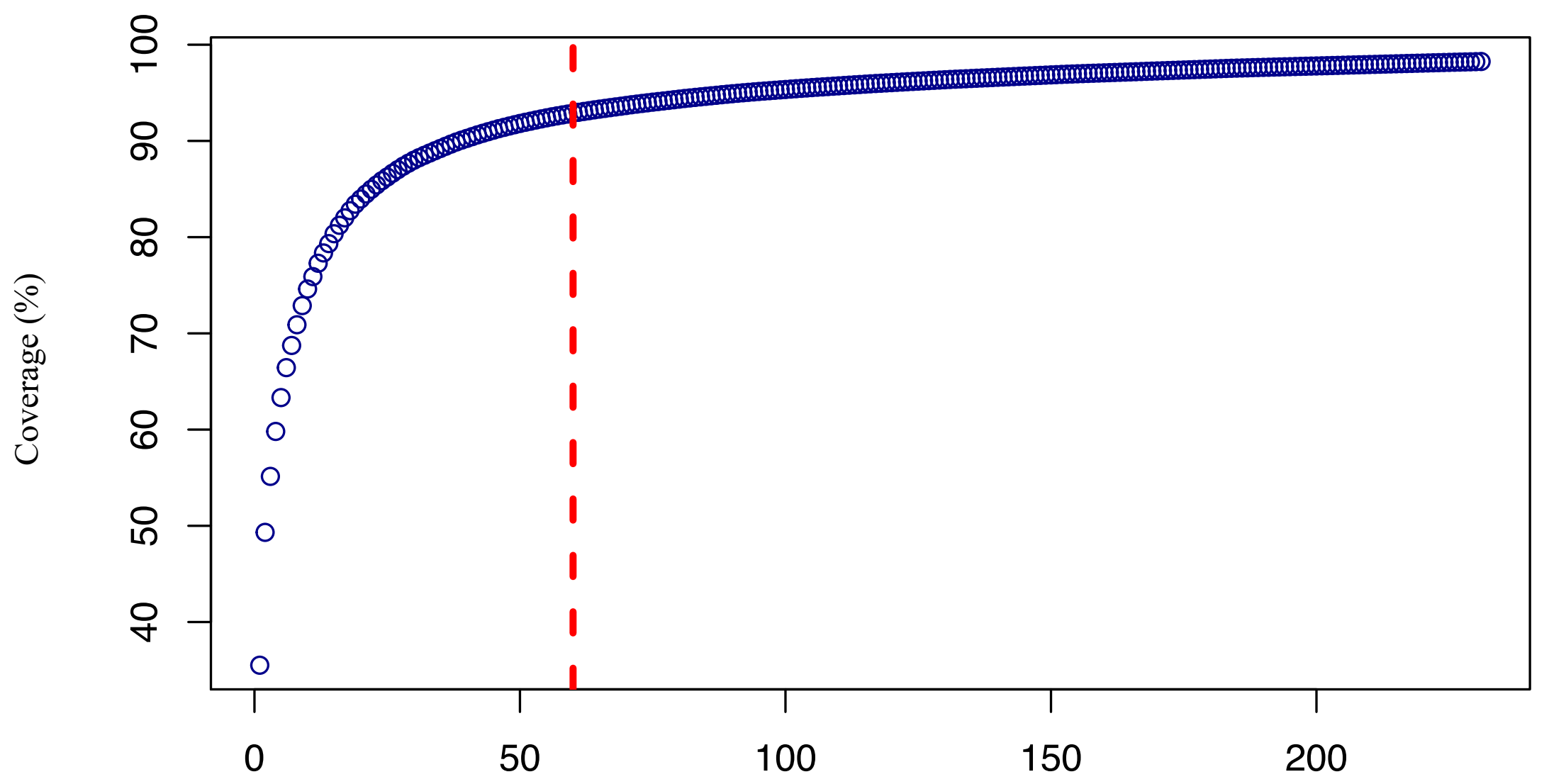

Number of samples 


\section{Parsed Citations}

Appels, R., Eversole, K., Feuillet, C., et al. 2018. Shifting the limits in wheat research and breeding using a fully annotated reference genome. Science, 361.

Google Scholar: Author Only Title Only Author and Title

Bashalkhanov, S., Pandey, M. \& Rajora, O. P. 2009. A simple method for estimating genetic diversity in large populations from finite sample sizes. BMC Genetics, 10, 84.

Google Scholar: Author Only Title Only Author and Title

Brandolini, A \& Heun, M. 2019. Genetics of brittleness in wild, domesticated and feral einkorn wheat (Triticum monococcum L.) and the place of origin of feral einkorn. Genetic Resources and Crop Evolution, 66, 429-439.

Google Scholar: Author Only Title Only Author and Title

Brandolini, A, Volante, A \& Heun, M. 2016. Geographic differentiation of domesticated einkorn wheat and possible Neolithic migration routes. Heredity, 117, 135-141.

Google Scholar: Author Only Title Only Author and Title

Browning, B. L., Zhou, Y. \& Browning, S. R. 2018. A One-Penny Imputed Genome from Next-Generation Reference Panels. The American Journal of Human Genetics, 103, 338-348.

Google Scholar: Author Only Title Only Author and Title

Brunazzi, A, Scaglione, D., Talini, R. F., et al. 2018. Molecular diversity and landscape genomics of the crop wild relative Triticum urartu across the Fertile Crescent. The Plant Journal, 94, 670-684.

Google Scholar: Author Only Title Only Author and Title

Charmet, G. 2011. Wheat domestication: Lessons for the future. Comptes Rendus Biologies, 334, 212-220.

Google Scholar: Author Only Title Only Author and Title

Chen, S., Guo, Y., Briggs, J., et al. 2018. Mapping and characterization of wheat stem rust resistance genes SrTm5 and Sr60 from Triticum monococcum Theoretical and Applied Genetics, 131, 625-635.

Google Scholar: Author Only Title Only Author and Title

Chen, W.-C. 2011. Overlapping codon model, phylogenetic clustering, and alternative partial expectation conditional maximization algorithm lowa State University.

Google Scholar: Author Only Title Only Author and Title

Comai, L. 2005. The advantages and disadvantages of being polyploid. Nature Reviews Genetics, 6, 836-846.

Google Scholar: Author Only Title Only Author and Title

Danecek, P., Auton, A, Abecasis, G., et al. 2011. The variant call format and VCFtools. Bioinformatics, 27, 2156-2158.

Google Scholar: Author Only Title Only Author and Title

Díez, M. J., De La Rosa, L., Martín, l., et al. 2018. Plant Genebanks: Present Situation and Proposals for Their Improvement. the

Case of the Spanish Network. Frontiers in Plant Science, 9.

Google Scholar: Author Only Title Only Author and Title

Dong, L., Huo, N., Wang, Y., et al. 2012. Exploring the diploid wheat ancestral Agenome through sequence comparison at the high-molecular-weight glutenin locus region. Molecular Genetics and Genomics, 287, 855-866.

Google Scholar: Author Only Title Only Author and Title

Dorofeev, V., Filatenko, A, Migušova, E., et al. 1979. Pšenica (Wheat). Kolos", Leningrad.

Google Scholar: Author Only Title Only Author and Title

Dvorak, J., Mcguire, P. E. \& Cassidy, B. 1988. Apparent sources of the Agenomes of wheats inferred from polymorphism in abundance and restriction fragment length of repeated nucleotide sequences. Genome, 30, 680-689.

Google Scholar: Author Only Title Only Author and Title

Dvořák, J., Terlizzi, P. D., Zhang, H.-B., et al. 1993. The evolution of polyploid wheats: identification of the Agenome donor species. Genome, 36, 21-31.

Google Scholar: Author Only Title Only Author and Title

Empilli, S., Castagna, R. \& Brandolini, A 2000. Morpho-agronomic variability of the diploid wheat Triticum monococcum L. Plant Genetic Resources Newsletter, 36-40.

Google Scholar: Author Only Title Only Author and Title

Endelman, J. B. 2011. Ridge Regression and Other Kernels for Genomic Selection with R Package rrBLUP. The Plant Genome, 4, 250-255.

Google Scholar: Author Only Title Only Author and Title 
Feldman, M. \& Levy, A A 2012. Genome evolution due to allopolyploidization in wheat. Genetics, 192, 763-774.

Google Scholar: Author Only Title Only Author and Title

Francis, R. M. 2017. pophelper: an R package and web app to analyse and visualize population structure. Molecular Ecology Resources, 17, 27-32.

Google Scholar: Author Only Title Only Author and Title

Fricano, A, Brandolini, A, Rossini, L., et al. 2014. Crossability of Triticum urartu and Triticum monococcum wheats, homoeologous recombination, and description of a panel of interspecific introgression lines. G3 (Bethesda, Md.), 4, 1931-1941.

Google Scholar: Author Only Title Only Author and Title

Gao, L., Koo, D.-H., Juliana, P., et al. 2021. The Aegilops ventricosa 2NvS segment in bread wheat: cytology, genomics and breeding. Theoretical and Applied Genetics, 134, 529-542.

Google Scholar: Author Only Title Only Author and Title

Glaubitz, J. C., Casstevens, T. M., Lu, F., et al. 2014. TASSEL-GBS: AHigh Capacity Genotyping by Sequencing Analysis Pipeline. PLOS ONE, 9, e90346.

Google Scholar: Author Only Title Only Author and Title

Goncharov, N. P. 2011. Genus Triticum L. taxonomy: the present and the future. Plant Systematics and Evolution, 295, 1-11.

Google Scholar: Author Only Title Only Author and Title

Harlan, J. R. \& Zohary, D. 1966. Distribution of Wild Wheats and Barley. Science, 153, 1074-1080.

Google Scholar: Author Only Title Only Author and Title

Heun, M., Schäfer-Pregl, R., Klawan, D., et al. 1997. Site of Einkorn Wheat Domestication Identified by DNAFingerprinting.

Science, 278, 1312-1314.

Google Scholar: Author Only Title Only Author and Title

Jeong, S., Kim, J.-Y., Jeong, S.-C., et al. 2017. GenoCore: A simple and fast algorithm for core subset selection from large genotype datasets. PLOS ONE, 12, e0181420.

Google Scholar: Author Only Title Only Author and Title

Kilian, B., Martin, W. \& Salamini, F. 2010. Genetic Diversity, Evolution and Domestication of Wheat and Barley in the Fertile Crescent. In: GLAUBRECHT, M. (ed.) Evolution in Action: Case studies in Adaptive Radiation, Speciation and the Origin of Biodiversity. Berlin, Heidelberg: Springer Berlin Heidelberg.

Google Scholar: Author Only Title Only Author and Title

Kilian, B., Özkan, H., Walther, A, et al. 2007. Molecular Diversity at 18 Loci in 321 Wild and 92 Domesticate Lines Reveal No Reduction of Nucleotide Diversity during Triticum monococcum (Einkorn) Domestication: Implications for the Origin of Agriculture. Molecular Biology and Evolution, 24, 2657-2668.

Google Scholar: Author Only Title Only Author and Title

Kolmer, J. A, Anderson, J. A \& Flor, J. M. 2010. Chromosome Location, Linkage with Simple Sequence Repeat Markers, and Leaf Rust Resistance Conditioned by Gene Lr63 in Wheat. Crop Science, 50, 2392-2395.

Google Scholar: Author Only Title Only Author and Title

Koo, D.-H., Liu, W., Friebe, B., et al. 2017. Homoeologous recombination in the presence of Ph1 gene in wheat. Chromosoma, 126, 531-540.

Google Scholar: Author Only Title Only Author and Title

Langmead, B., Trapnell, C., Pop, M., et al. 2009. Ultrafast and memory-efficient alignment of short DNA sequences to the human genome. Genome biology, 10, 1-10.

Google Scholar: Author Only Title Only Author and Title

Li, O., Zhao, Y.-Y., Guo, N., et al. 2009. Effects of sample size and loci number on genetic diversity in wild population of grass carp revealed by SSR.

Ling, H.-Q., Ma, B., Shi, X., et al. 2018. Genome sequence of the progenitor of wheat A subgenome Triticum urartu. Nature, 557, 424-428.

Google Scholar: Author Only Title Only Author and Title

Mac Key, J. 1954. The taxonomy of hexaploid wheat. Svensk Bot Tidskr, 48, 579-590.

Google Scholar: Author Only Title Only Author and Title

Mac Key, J. 2005a. Wheat: its concept, evolution and taxonomy. Durum wheat breeding. Current approaches and future strategies, 1, 3-61.

Google Scholar: Author Only Title Only Author and Title

Mac Key, J. 2005b. Wheat: its concept, evolution, and taxonomy. Durum Wheat Breeding. CRC Press.

Google Scholar: Author Only Title Only Author and Title 
Middleton, C. P., Senerchia, N., Stein, N., et al. 2014. Sequencing of chloroplast genomes from wheat, barley, rye and their relatives provides a detailed insight into the evolution of the Triticeae tribe. PloS one, 9, e85761-e85761.

Google Scholar: Author Only Title Only Author and Title

Mondal, S., Rutkoski, J. E., Velu, G., et al. 2016. Harnessing Diversity in Wheat to Enhance Grain Yield, Climate Resilience, Disease and Insect Pest Resistance and Nutrition Through Conventional and Modern Breeding Approaches. Frontiers in Plant Science, 7.

Google Scholar: Author Only Title Only Author and Title

Nair, K. P. 2019. Utilizing crop wild relatives to combat global warming. Advances in Agronomy. Elsevier.

Google Scholar: Author Only Title Only Author and Title

Nei, M. 1973. Analysis of Gene Diversity in Subdivided Populations. Proceedings of the National Academy of Sciences, 70, 33213323.

Google Scholar: Author Only Title Only Author and Title

Nei, M. 1987. Molecular Evolutionary Genetics Columbia University Press New York 512.

Nicholson, G., Smith, A V., Jónsson, F., et al. 2002. Assessing population differentiation and isolation from single-nucleotide polymorphism data. Journal of the Royal Statistical Society: Series B (Statistical Methodology), 64, 695-715.

Google Scholar: Author Only Title Only Author and Title

Özkan, H., Tuna, M., Kilian, B., et al. 2010. Genome size variation in diploid and tetraploid wild wheats. AoB PLANTS, 2010.

Google Scholar: Author Only Title Only Author and Title

Paradis, E. \& Schliep, K. 2019. ape 5.0: an environment for modern phylogenetics and evolutionary analyses in R. Bioinformatics, 35, 526-528.

Google Scholar: Author Only Title Only Author and Title

Pintus, E., Sorbolini, S., Abera, A, et al. 2014. Use of locally weighted scatterplot smoothing (LOWESS) regression to study selection signatures in Piedmontese and Italian Brown cattle breeds. Anim Genet, 45, 1-11.

Google Scholar: Author Only Title Only Author and Title

Poland, J., Endelman, J., Dawson, J., et al. 2012b. Genomic selection in wheat breeding using genotyping-by-sequencing. The Plant Genome, 5.

Google Scholar: Author Only Title Only Author and Title

Poland, J. A, Brown, P. J., Sorrells, M. E., et al. 2012a. Development of high-density genetic maps for barley and wheat using a novel two-enzyme genotyping-by-sequencing approach. PloS one, 7, e32253-e32253.

Google Scholar: Author Only Title Only Author and Title

Porto-Neto, L. R., Lee, S. H., Lee, H. K., et al. 2013. Detection of Signatures of Selection Using FST. In: GONDRO, C., VAN DER WERF, J. \& HAYES, B. (eds.) Genome-Wide Association Studies and Genomic Prediction. Totowa, NJ: Humana Press.

Google Scholar: Author Only Title Only Author and Title

Pourkheirandish, M., Dai, F., Sakuma, S., et al. 2018. On the Origin of the Non-brittle Rachis Trait of Domesticated Einkorn Wheat. Frontiers in Plant Science, 8.

Google Scholar: Author Only Title Only Author and Title

Raj, A, Stephens, M. \& Pritchard, J. K. 2014. fastSTRUCTURE: Variational Inference of Population Structure in Large SNP Data Sets. Genetics, 197, 573-589.

Google Scholar: Author Only Title Only Author and Title

Rouse, M. N. \& Jin, Y. 2011. Stem Rust Resistance in AGenome Diploid Relatives of Wheat. Plant Disease, 95, 941-944.

Google Scholar: Author Only Title Only Author and Title

Saintenac, C., Zhang, W., Salcedo, A, et al. 2013. Identification of Wheat Gene Sr35 That Confers Resistance to Ug99 Stem Rust Race Group. Science, 341, 783-786.

Google Scholar: Author Only Title Only Author and Title

Schiemann, E. 1948. Weizen. Roggen, Gerste: Systematik Geschichte und Verwendung, Verlag Gustav Fischer, Jena, Germany. Google Scholar: Author Only Title Only Author and Title

Sheedy, J. G., Thompson, J. P. \& Kelly, A 2012. Diploid and tetraploid progenitors of wheat are valuable sources of resistance to the root lesion nematode Pratylenchusthornei. Euphytica, 186, 377-391.

Google Scholar: Author Only Title Only Author and Title

Singh, N., Wu, S., Tiwari, V., et al. 2019. Genomic Analysis Confirms Population Structure and Identifies Inter-Lineage Hybrids in Aegilops tauschii. Frontiers in Plant Science, 10.

Google Scholar: Author Only Title Only Author and Title 
Van Slageren, M. W. 1994. Wild wheats: a monograph of Aegilops L. and Amblyopyrum (Jaub. \& Spach) Eig (Poaceae). Wageningen Agricultural University Papers.

Google Scholar: Author Only Title Only Author and Title

Van Zeist, W. 1992. The origin and development of plant cultivation in the Near East. Nichibunken Japan Review, 149-165. Google Scholar: Author Only Title Only Author and Title

Wang, X., Luo, G., Yang, W., et al. 2017. Genetic diversity, population structure and marker-trait associations for agronomic and grain traits in wild diploid wheat Triticum urartu. BMC Plant Biology, 17, 112.

Google Scholar: Author Only Title Only Author and Title

Weir, B. S. \& Cockerham, C. C. 1984. Estimating F-statistics for the analysis of population structure. evolution, 1358-1370.

Google Scholar: Author Only Title Only Author and Title

Wulff, B. B. H. \& Moscou, M. J. 2014. Strategies for transferring resistance into wheat: from wide crosses to GM cassettes.

Frontiers in Plant Science, 5.

Google Scholar: Author Only Title Only Author and Title

Zaharieva, M. \& Monneveux, P. 2014. Cultivated einkorn wheat (Triticum monococcum L. subsp. monococcum): the long life of a founder crop of agriculture. Genetic Resources and Crop Evolution, 61, 677-706.

Google Scholar: Author Only Title Only Author and Title 